\title{
Correferencialidade sintática e alinhamento em Xikrín do Cateté
}

\author{
Lucivaldo Silva da Costa ${ }^{1}$ \\ Bep Nhõrõ-Ti Xikrín ${ }^{2}$ \\ Ana Suelly Arruda Câmara Cabral ${ }^{3}$
}

\section{Resumo}

Neste artigo descrevemos as principais estratégias usadas por falantes Xikrín para expressar referência compartilhada ou disjunta através de fronteiras de orações unidas por parataxe ou por hipotaxe. Xikrín do Cateté é uma variedade de uma das línguas Jê falada no estado do Pará. Outras variedades dessa língua são o Kayapó e o Mẽbengokre. Nós tratamos também de mostrar padrões congruentes de alinhamentos internos à oração e alinhamentos através de orações. Nossa análise beneficiou-se das contribuições de Foley e Van Valin (1984) e Dixon (1994) quanto a switch-reference e functional reference através de diferentes línguas, assim como do estudo sobre ergatividade em Xokléng de autoria de Urban (1985).

Palavras-chave: Sistemas de alinhamento, alinhamento sintático, combinação de orações, correferência, língua Xikrín, família Jê.

\begin{abstract}
In this paper we describe the main strategies used by Xikrin speakers to express shared reference or disjunctive reference across clauses junctures, in which the clauses are in a parataxis or hypotaxis relation. Xikrín of Cateté is a variety of a Jê language spoken in Pará state. Other varieties of the same language are called Kayapó or Mebengokré. We are also concerned with showing congruity patterns involving clause internal and clause external alignment. Our analysis of the Xikrin data has benefited from Foley and Van Valin (1984) and Dixon (1994) insights on switch-reference and functional reference across different languages, as well from Urban (1985) on ergativity in Xokléng.
\end{abstract}

Keywords: Alignment systems, syntactic alignment, clause combining, co-reference, Xikrín language, Jê family.

\footnotetext{
${ }^{1}$ Professor na Universidade Federal do Pará, Campus de Marabá e pesquisador do Laboratório de Línguas Indígenas da Universidade de Brasília.

${ }^{2}$ Professor Xikrín da Escola Indígena Bepkaroti Xikrín.

${ }^{3}$ Professora do Departamento de Linguística do Instituto de Letras, Vice-Coordenadora do Laboratório de Línguas Indígenas e Coordenadora do Núcleo de Estudos da Amazônia do Centro de Estudos Avançados Multidisciplinares da Universidade de Brasília.
} 


\section{Introdução}

Neste artigo descrevemos estratégias da língua Xikrín do Cateté para marcar referência compartilhada ou disjunta de argumentos através de orações combinadas por parataxe ou por hipotaxe. ${ }^{4} \mathrm{O}$ Xikrín do Cateté é uma das variedades do Mẽbengokré, que por sua vez constitui uma das línguas do ramo Setentrional da família Jê (na hipótese em desenvolvimento por Rodrigues $(1986,1999,2001,2009)$ sobre um tronco linguístico MacroJê, a família Jê é um dos 12 sub-ramos que constituem este agrupamento genético). As outras variedades do Mẽbengokré são o Txukahamãj, o Kayapó do Gorotire, o Kararaõ, o Kokraimóro, o Kubenkrangnotí, o Menkrangnotí, o Kubenkrankégn e o Xikrín do Bakajá. As demais línguas do ramo Jê Setentrional são o complexo Timbíra, o Apinajé, o Suyá o Tapayúna ou Kajkwakratxí e o Panará.

O objetivo deste estudo é identificar as correlações entre o sistema de alinhamento intraoracional existente na língua, que é caracterizado por uma cisão em que um sistema nominativo-absolutivo alterna com um sistema ergativo-absolutivo (Cabral, Rodrigues e Costa [2001] 2003; Costa 2003), com padrões de alinhamento acionados na combinação de orações. Será mostrado que a língua Xikrín, na combinação de orações por parataxe, manifesta uma cisão: em certos contextos é acionado um padrão nominativo e, em outros, um padrão misto, nominativo e absolutivo. Esses padrões são monitorados por um sistema de switch-reference (ou referência alternada) que sinaliza por meio de diferentes estratégias morfossintáticas quando o referente de um argumento $\mathrm{A}, \mathrm{S}$ ou $\mathrm{O}$ de uma oração dependente é correferente ou não com um argumento da oração principal. Identificamos, assim, os padrões combinatórios de correferência igual ou disjunta e identificamos correlações destes padrões com os padrões de alinhamento que ocorrem no interior de orações, observando o que têm em comum e sua importância para a compreensão de aspectos importantes da organização da gramática Xikrín.

\footnotetext{
${ }^{4}$ Abreviaturas: ACT. $=$ active; CONJ. $=$ conjunção; CONT $=$ continuativo; CORREF COREF. $=$ correferencial; DEF. $=$ definido; $\mathrm{DIR}=$ direcional; $\mathrm{ENF}=$ enfático; $\mathrm{ERG}=$ ergativo; $\mathrm{FEM} .=$ feminino; IRLS = irrealis; LOC = locativo; $\mathrm{MS}=$ mesmo sujeito; $\mathrm{NEG}=$ negação; NLZ = nominalizador; $\mathrm{NOM}$ $=$ NOMINATIVO $;$ ONC = objeto não contíguo; PAS = passado; PAST $=$ passado; PAUC $=$ paucal; PL $=$ plural; $3 \mathrm{PLU}=$ plural; $\mathrm{POSP}=$ posposição $; \mathrm{R}^{1}-=$ relacional que marca a contiguidade de um determinante; $\mathrm{R}^{4}-$ = relacional que marca em um núcleo um determinante genérico (e/ou humano); $\mathrm{RLS}=$ realis; $\mathrm{SG} .=$ singular; $\mathrm{STAT} .=$ estativo; $\mathrm{SS}=$ mesmo sujeito; $1 \mathrm{SG}=$ primeira do singular; $1 \pm 3$ = primeira pessoa ou nós exclusivo; $2 \pm 3=$ você ou vocês.
} 
Switch-reference foi estudado pela primeira vez, no contexto das línguas Macro-Jê, por Urban (1985), em Ergativity and accusativity in Shokleng $(G E)$. Esta foi também a descrição mais ampla do fenômeno em tela até o presente no contexto das línguas da família Jê. O mesmo fenômeno foi identificado posteriormente em outras línguas da família: no Krahô por Popjes e Popjes (1986), no Parkatejê por Ferreira (2003), no Canela Apãniekrá por Alves (2004).

Os dados do Xikrín também evidenciam a presença de um sistema de switch-reference como esperado, dada a sua proximidade com as demais línguas supramencionadas. Entretanto, nele o fenômeno não pode ser visto como uma simples marcação de correferência igual ou disjunta entre A ou S de duas orações, mas como um sistema mais complexo, próximo do que foi descrito para o Xokléng por Urban (1985). Em Xikrín do Cateté, assim como em Xokléng, o fenômeno switch-reference observa variáveis gramaticais como transitividade, mas diferentemente deste, regula-se também por outros princípios que constituem a gramática Xikrín, como o que determina a nominalização de predicados verbais em contextos nos quais funcionam como nomes adjetivos (ou de qualidade) ou substantivos (Cabral, Rodrigues e Costa [2001] 2003; Costa 2003).

Nas duas seções seguintes, apresentamos considerações sobre as características e variações de sistemas de correferência identificados por Foley e Van Valin (1984) e por Dixon (1994) - switch-reference e functional reference (referência funcional) - em línguas geneticamente diferentes e geograficamente distantes, visto que os resultados das abordagens funcional e tipológica desses fenômenos feitas por esses autores contribuem com previsões e generalizações acerca de princípios gerais que acionam sistemas correferenciais através das línguas.

Nas seções posteriores, retomamos as análises de Cabral, Rodrigues e Costa ([2001] 2003) e de Costa (2003), as quais descrevem o Xikrín como um tipo de língua que apresenta em seu escopo intraoracional uma cisão de alinhamento peculiar, o qual, em certas situações é nominativo-absolutivo, mas em outras é ergativo-absolutivo. Mostramos, em seguida, que as combinações de orações por parataxe refletem essa mesma organização no seu sistema de correferência - nominativo em certas circunstâncias e absolutivo em outras, mas, em situações específicas, cruzando os dois tipos de alinhamento no escopo único da combinação de duas orações.

\footnotetext{
${ }^{5}$ A primeira versão desse estudo foi apresentada durante o II Macro-Jê, na Universidade Estadual de Campinas, em 2001.
} 


\section{Switch-reference e Functional reference}

Consideramos aqui dois dos tipos de sistemas de coesão discursiva discutidos por Foley e Van Valin (1984) e por Dixon (1994), switch-reference e functional reference, por serem os tipos relevantes de correferência para a análise dos dados do Xikrín.

\subsection{Correferencialidade em junções de orações ou interoracionais}

Foley e Van Valin (1984) ao tratar dos sistemas de coesão discursiva, observam que o nível de junturas periféricas - união de construções com núcleos, argumentos e elementos periféricos (expressões adverbiais) próprios - difere do nível de junturas de núcleos - em que se unem construções com núcleos e argumentos próprios, mas que compartilham os mesmos elementos periféricos-, visto que, nesteúltimo, há fortes restrições de correferencialidade entre argumentos dos núcleos individuais que constituem a juntura. Quanto ao nível da juntura de núcleos que formam um núcleo complexo - núcleos que compartilham tanto o mesmo argumento quanto elementos periféricos, como nas construções de verbos seriais de línguas africanas -, há obrigatoriedade de correferência entre todos os membros da junção. Foley e Van Valin (op.cit., p. 193) ilustram as restrições de correferência entre junturas de núcleos de núcleo complexo, com dados do Akan disponibilizados em Schachter (1974). O exemplo em (5.8) abaixo é gramatical, pois os referentes dos argumentos dos dois núcleos são correferentes, mas o exemplo (5.9) é agramatical por não haver correferência entre os atores (argumentos agentes):

$\begin{array}{llll}\text { (5.8) } & \text { kofi yec adwuma maa Amma } \\ \text { Kofi did work } & \text { gave Amma }\end{array}$

'Kofi did work for Amma' (Foley e Van Valin 1984:193)

(5.9) *kofi yec adwuma Kwaku maa Amma

Kofi did work Kwaku gave Amma

'Kofi did work Kwaku gave Amma' (Foley e Van Valin 1984:193)

O exemplo (5.9) é agramatical, pois o sujeito de gave deve ser correferente com o sujeito de did work.

Foley e Van Valin (1984:194) observam que outras línguas são menos restritivas, permitindo que junturas nucleares sejam formadas com correferência entre U(ndergoer)-A(gent) ou U(ndergoer)-S(subject), como em Barai (Olson 1991): 

(5.11) na ki-ia bu va-e
$1 \mathrm{SG}$ say-3PLU 3PL go-PAST
'I spoke to them; (causing) they went' or 'I made them go'
(Foley e Van Valin 1984:194)

Os autores (p.195) observam também que, por junturas periféricas serem formadas pela combinação de periferias, cada uma com seus próprios núcleos, argumentos nucleares e elementos periféricos (adverbiais), não se espera que haja restrições de correferência entre os argumentos nucleares na formação de junções, mas ressaltam que, nessas situações, a determinação de correferencialidade entre constituintes pode ser problemática, razão pela qual as línguas desenvolveram várias estratégias para resolver impasses. Foley e Van Valin (op.cit., p.196) apresentam outras diferenças entre as junturas de núcleos e de constituintes de núcleos complexos e junturas de construções periféricas: nas primeiras, não há marcação individual de tempo e modo, mas compartilhada, e são marcadas apenas por justaposição, ao passo que as junturas periféricas permitem a existência de várias relações semânticas entre as junturas, e as línguas tendem a ter um largo inventário de morfemas para expressar tais relações.

Todas essas diferenças justificam, segundo os autores, a necessidade de monitoramento de correferência entre os argumentos em junções periféricas. Segundo Foley e Van Valin (1984:321), em virtude do alto índice de relações semânticas que podem ser expressas em junturas periféricas, elas são comuns em diferentes tipos de discurso, de forma que o monitoramento de correferência de argumentos nucleares através dessas junturas é uma função central das gramáticas de toda língua. Ressaltam que o uso de sintagmas nominais para se referirem a todos os participantes em cada juntura seria uma solução para o problema, mas não a solução real, dada a tendência pervasiva das línguas de omitir ou pronominalizar informações dadas ou tópicas, de forma que o problema da identificação de participante é um problema real.

Foley e Van Valin (op.cit::322) postulam a existência de quatro sistemas básicos para marcar correferência através das línguas e, segundo eles, uma língua pode empregar um ou mais de um desses sistemas: a) a combinação de pivots pragmáticos com oposição de voz, b) referência alternada, c) gênero como elemento acionador de correferência, e d) variáveis sociolinguísticas como base de correferência. Segundo esses atores, mecanismos em a) são encontrados em línguas como o Inglês, e correspondem ao sistema de função alternada, exemplificada pelos exemplos que reproduzimos abaixo, numerados de acordo com o texto original (op.cit.:322): 
(7.1) a. Fred wants to see Marsha.

b. *Fred wants Marsha to see [him].

(7.2) a. Max persuaded Fred to see Marsha.

b. *Max persuaded Fred Marsha to see [him].

(7.3) a. Oscar went to the store and spoke to Bill

b. *Oscar went to the store and Bill spoke to [him].

Como observam Foley e Van Valin (1984:322), em (7.1a) existe correferência entre $P$ (ivots) $\operatorname{Pr}$ (agmáticos) PrP-PrP, e em (7.2a) há correferência entre U-PrP, e em cada caso o PrP na oração coordenada é zero; já os exemplos correspondentes em $b$ são agramaticais, visto que existe correferência entre PrP-U e U-U, em que $U$ na oração coordenada ocorre como zero em uma posição de argumento nuclear não pivot (objeto direto). Assim, para que um argumento da oração coordenada seja omitido, teria que ser potencialmente um PrP, o que só é possível com uma construção passiva:

(7.4) a. Fred wants to be seen by Marsha.

b. Max persuaded Fred to be seen by Marsha.

c. Oscar went to the store and was spoken to by Bill.

Foley e Van Valin (op.cit.) reiteram que em todos os casos, o argumento zero-pronominalizado deve ser o PrP de sua oração, de forma que a oposição ativa-passiva desempenha importante papel nesse sistema. Os autores se referem a esse tipo de sistema switch function (função alternada).

O outro sistema que é pertinente para o estudo de correferencialidade em Xikrin é o sistema de switch-reference em que a morfologia verbal indica se um SN na primeira oração é correferencial com um NP particular da oração seguinte. Segundo esses autores a morfologia da referência alternada sinaliza a correferência ou a não correferência entre S/A SmP-S/A, embora outras línguas operem em outras bases. ${ }^{6}$

\footnotetext{
${ }^{6}$ Os outros dois tipos de sistemas de manutenção de correferência - o sistema de gênero e o sistema de referência discursiva - não se manifestam em Xikrín. O Primeiro envolve a codificação morfológica aberta de classificação de sintagma nominais, ao passo que o segundo faz uso sutil de variáveis sociolinguísticas, mas a sinalização não se dá diretamente nas formas linguísticas, como ocorre, segundo Foley e Van Valin, na fala honorífica em Japonês e Coreano, ou no caso das regras de inferência conversacional baseada em conhecimento cultural existente em outras línguas.
} 


\subsection{Correferencialidade e restrições de alinhamento}

Dixon (1994), ao tratar de ergatividade sintática interoracional em diferentes línguas, propõe que algumas delas impõem duas restrições com respeito à função sintática ( $\mathrm{S}$ ou $\mathrm{A}$ ou $\mathrm{O})$ dos sintagmas nominais correferenciais. Dessas restrições, uma trata como equivalentes $\mathrm{S}$ e A, enquanto outra trata como equivalentes $\mathrm{S}$ e O. Essa equivalência é descrita pela categoria de pivot (termo usado pela primeira vez por Dixon 1979). Essa categoria tem, segundo Dixon (1994:143), duas variedades, e as línguas podem fazer uso de uma das duas variedades ou de uma variedade mista:

S/A - o SN correferencial deve estar em uma função de S ou de A derivada em cada uma das orações a serem combinadas.

$\mathrm{S} / \mathrm{O}$ - o SN correferencial deve estar em uma função de $\mathrm{S}$ ou de $\mathrm{O}$ derivada em cada uma das orações a serem combinadas.

Dixon (op. cit. p. 154) postula que em línguas com referência alternada, um segundo SN pode ser omitido facilmente sem possibilidade de ambiguidade e confusão, sem que sejam requeridas operações sintáticas de passiva ou de antipassiva, uma vez que não há necessidade de transformar um $\mathrm{SN}$, O ou A em S para facilitar a omissão do NP sem chance de ambiguidade. Como enfatiza Dixon (op. cit.), passivas e antipassivas derivam uma oração intransitiva de uma transitiva, a passiva coloca um $\mathrm{O}$ subjacente em uma função de $S$, ao passo que uma antipassiva coloca $A$ em função de $S$ e rebaixa o objeto, que muitas vezes é omitido. E línguas com sistema de referência alternada não teriam passiva nem antipassiva.

Dixon (1994) considera apenas três sistemas com implicações sintáticas para alinhamento: referência alternada, restrição de pivot e línguas que não apresentam nem referência alternada nem restrição de pivot. Nesse último tipo de língua, segundo Dixon (op. cit.), qualquer tipo de oração pode ser combinado por coordenação ou por subordinação, desde que essa combinação seja semanticamente aceita.

\section{Uma nota sobre alinhamento intrassentencial em Xikrín}

Antes de abordarmos manifestações de correferencialidade interoracional em Xikrín, discorremos, de forma resumida, sobre os padrões de alinhamento intraoracionais nessa língua, com base em Cabral, Rodrigues e Costa ([2001] 2003) e em Costa (2003). O Xikrín é uma língua que apresenta uma cisão em seu alinhamento: um padrão ergativo-absolutivo ocorre nas 
situações em que uma oração tem como núcleo uma forma nominalizada de um verbo - em função de adjetivo, de substantivo, de advérbio (Cabral, Rodrigues e Costa [2001] 2003; Costa 2003), e um padrão nominativoabsolutivo ocorre nas orações com núcleo verbal processual (Cabral, Rodrigues e Costa [2001] 2003; Costa 2003). O Xikrín possui duas séries de marcadores pessoais, A e B:

\begin{tabular}{|c|c|c|}
\hline & Série A & Série B \\
\hline $1 \pm 3$ & $b a$ & $i$ \\
\hline $1+2$ & $g u$ & $g u b a$ \\
\hline $2 \pm 3$ & $g a$ & $a$ \\
\hline
\end{tabular}
Quadro de marcadores pessoais do Xikrín
(Cabral, Rodrigues e Costa [2001] 2003:23)

Os marcadores da série $B$, sem nenhum elemento adicional, correspondem a referentes singulares ou duais. Combinam-se com as partículas paucal ar(i), que indica três ou poucos referentes, e com a partícula plural $m \tilde{e}$, que indica muitos referentes. As formas dessa série ocorrem como possuidor (ex. 1), objeto de posposição (ex. 2), objeto direto (ex. 3), sujeito de predicados descritivos ${ }^{7}$ (ex. 4), assim como sujeito de verbos intransitivos de orações modificadas por uma expressão adverbial (ex. 5 e 6). A ocorrência desses marcadores nessas funções caracteriza-os como marcadores pessoais absolutivos.
1) $\begin{array}{ll}i & n-i k r a \\ 1 \pm 3 & \mathrm{R}^{1}-\mathrm{mão}\end{array}$
'minha mão ou mão de nós dois (eu e ele)'
(Cabral, Rodrigues e Costa [2001] 2003:23)

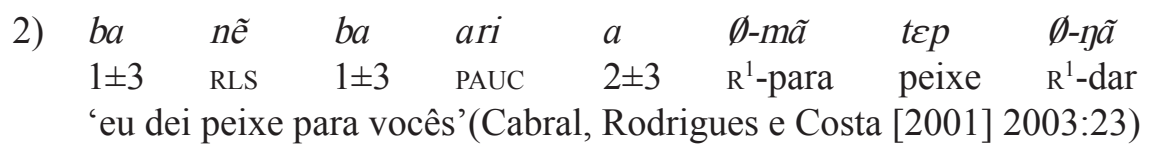

3) $\begin{array}{lllll}b a & n \tilde{e} & b a & a & \emptyset \text {-mũ } \\ 1 \pm 3 & \text { RLS } & 1 \pm 3 & 2 \pm 3 & \mathrm{R}^{1} \text {-ver }\end{array}$

'eu vi você'(Cabral, Rodrigues e Costa [2001] 2003:24)

${ }^{7}$ Predicados descritivos têm por núcleo nomes de qualidade. 
4) $\quad a \quad \emptyset-m \varepsilon j \quad$ kumrẽ j

$2 \pm 3 \quad \mathrm{R}^{1}$-ser.bom mesmo

'você é bom mesmo' (Cabral, Rodrigues e Costa [2001] 2003:24)

5) $\quad$ ga $\quad$ a $\quad$ Ø-to-ro $\quad$ ket

$2 \pm 3 \quad 2 \pm 3 \quad \mathrm{R}^{1}$-dançar-NLZ NEG

'você não dançou'(Cabral, Rodrigues e Costa [2001] 2003:27)

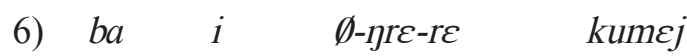

$1 \pm 3 \quad 1 \pm 3 \quad \mathrm{R}^{1}$-cantar-NLZ muito

'eu canto muito'(Cabral, Rodrigues e Costa [2001] 2003:27)

Os marcadores da série A ocorrem como sujeitos de verbos transitivos e intransitivos (exs. 7 e 8), e são, portanto, marcadores pessoais nominativos. Podem também ocorrer como pronomes enfáticos, em coocorrência com eles mesmos (ex.7) ou com os da série B (exemplo 9). As partículas ari 'paucal' e $m$ ẽ 'plural' são antepostas aos marcadores enfáticos (ex. 9 e 10) e pospostas aos nominativos (ex. 9 e 10) para indicar poucos ou muitos elementos, respectivamente.

7) $b a \quad$ nẽ $\quad b a \quad m e \tilde{-}-m \tilde{u}$

$1 \pm 3$ ENF RLS $1 \pm 3 \quad \mathrm{R}^{3}$-ver

'eu vi gente' (Costa, notas de campo, 2008)

8) $g a \quad n \tilde{e} \quad g a \quad b o j$

$2 \pm 3 \quad$ RLS $\quad 2 \pm 3 \quad$ chegar

'vocês chegaram'(Cabral, Rodrigues e Costa [2001] 2003:24)

9) ari ga ñ $g a$ ari boj

PAU $2 \pm 3$ RLS $2 \pm 3$ PAU chegar

'vocês chegaram'(Costa, notas de campo, 2008)

10) $m \tilde{e} \quad b a$ ñ $\quad b a \quad m \tilde{e} \quad i \quad \emptyset$-krãkats

PL $\quad 1 \pm 3 \quad$ RLS $\quad 1 \pm 3 \quad$ pl $\quad 1 \pm 3 \quad \mathrm{R}^{1}$-acordar

'nós acordamos'(Costa, notas de campo, 2008)

A língua Xikrín manifesta, assim, dois tipos básicos de alinhamento de suas formas pronominais, um padrão nominativo-absolutivo e outro ergativoabsolutivo. O sujeito de um verbo transitivo, seja este bivalente ou trivalente, é expresso pela mesma série nominativa (série A) usada para expressar o sujeito dos intransitivos processuais, ao passo que seu objeto se exprime 
pelos marcadores absolutivos (série B), tal como o sujeito de predicados intransitivos descritivos e processuais quando o contexto sintático requer uma forma nominalizada do verbo. Configura-se, assim, com respeito aos transitivos, um padrão nominativo-absolutivo, que pode ser observado nos seguintes exemplos:

\section{Predicados com núcleos transitivos bivalentes}
11) $b a \quad$ nẽ $\quad b a \quad$ warikoks $\emptyset$-bi
$1 \pm 3 \quad$ RLS $\quad 1 \pm 3 \quad$ cachimbo $\quad \mathrm{R}^{1}$-pegar
'eu peguei o cachimbo'(Cabral, Rodrigues e Costa [2001] 2003:25)

12) $\begin{array}{lllll}b a & d 3 a & b a & \text { kwərə } & j \text {-are } \\ 1 \pm 3 & \text { RLS } & 1 \pm 3 & \text { mandioca } & \mathrm{R}^{1} \text {-arrancar }\end{array}$

'eu arrancarei mandioca' (Cabral, Rodrigues e Costa [2001] 2003:25)

\section{Predicados com núcleos transitivos trivalentes}

13) $b a \quad n \tilde{e} \quad b a \quad$ a $\quad \emptyset$-kra $\quad \emptyset$-ma $\quad$ tep $\quad \emptyset-\eta \tilde{a}$ $1 \pm 3 \quad$ RLS $\quad 1 \pm 3 \quad 2 \pm 3 \quad \mathrm{R}^{1}$-filho $\mathrm{R}^{1}$-para peixe $\mathrm{R}^{1}$-dar 'eu dei peixe para o filho de você'(Cabral, Rodrigues e Costa [2001] 2003:25)

14) $b a \quad$ ne $\quad b a \quad k u-m \tilde{a} \quad k a p r a n$ j-ano $1 \pm 3 \quad$ RLS $1 \pm 3 \quad \mathrm{R}^{2}$-para jabuti $\quad \mathrm{R}^{1}$-mandar 'eu mandei jaboti para ele'(Cabral, Rodrigues e Costa [2001] 2003:25)

\section{Predicados com núcleos intransitivos monovalentes descritivos}

15) $i \quad \emptyset$-mej kumrẽj

$1 \pm 3 \quad \mathrm{R}^{1}$-ser.bom mesmo

'eu sou bom mesmo'(Cabral, Rodrigues e Costa [2001] 2003:26)

16) $g a \quad$ ก $\quad$ ga $a \quad \emptyset$-mej kumrẽj

$2 \pm 3 \quad$ RLS $\quad 2 \pm 3 \quad 2 \pm 3 \quad \mathrm{R}^{1}$-ser.bom mesmo

'você é boa mesmo'(Cabral, Rodrigues e Costa [2001] 2003:26)

\section{Intransitivos monovalentes processuais}

17) $b a \quad$ நо̃rõ

$1 \pm 3$ dormir 'eu durmo'
18) $b a \quad d 3 a \quad b a \quad$ тиa $1 \pm 3 \quad$ IRLS $1 \pm 3$ chorar 'eu vou chorar'(Cabral, Rodrigues e Costa [2001] 2003:26) 


\section{Intransitivos bivalentes}

19) $b a \quad i \quad \emptyset$-ma $\quad m \tilde{e} \quad a \quad \emptyset-k \tilde{I}$

$1 \pm 3 \quad 1 \pm 3 \quad \mathrm{R}^{1}$-para $\quad$ PL $\quad 2 \pm 3 \quad \mathrm{R}^{2}$-gostar

'eu gosto de vocês'(Cabral, Rodrigues e Costa [2001] 2003:26)

20) $b a \quad i \quad \emptyset$-ma menire $\emptyset$-kĩ

$1 \pm 3 \quad 1 \pm 3 \quad \mathrm{R}^{1}$-para mulher $\mathrm{R}^{2}$-gostar

'eu gosto da mulher'(Cabral, Rodrigues e Costa [2001] 2003:26)

Em Xikrín, no padrão ergativo-absolutivo, o sujeito dos verbos transitivos é expresso pelos marcadores pessoais da série B regidos pela posposição -je do caso ergativo, enquanto o objeto é expresso por marcador dessa mesma série contíguo ao verbo ${ }^{8}$. Exemplos:

21) $\begin{array}{llllll}g a & a & \emptyset-j \varepsilon & i & j-o k-\emptyset & k e t\end{array}$

$2 \pm 3 \quad 2 \pm 3 \quad \mathrm{R}^{1}$-ERG $\quad 1 \pm 3 \quad \mathrm{R}^{1}$-pintar-NLZ $\quad$ NEG

'você não me pintou'(Cabral, Rodrigues e Costa [2001] 2003:27)

22) $\begin{array}{llllllll}g a & n \tilde{\mathrm{e}} & g a & a & \emptyset-j \varepsilon & \text { tiriti } & \emptyset \text {-krẽ-n } & \text { pram } \\ 2 \pm 3 & \mathrm{RLS} & 2 \pm 3 & 2 \pm 3 & \mathrm{R}^{1} \text { - ERG } & \text { banana } & \mathrm{R}^{1} \text {-comer-NLZ } & \text { quere }\end{array}$ 'você quer comer banana'(Cabral, Rodrigues e Costa [2001] 2003:27)

23) $i-j \varepsilon \quad m \tilde{e}-\eta r \varepsilon-r \varepsilon \quad m a-r i \quad k e t$

$1 \pm 3$-ERG $\mathrm{R}^{3}$-cantar-NLZ saber- NLZ NEG

'eu não sei cantar'(Costa, notas de campo 2008)

Quando os predicados intransitivos processuais são modificados por palavra adverbial, ocorrem nominalizados e o determinante deles é marcado pelas formas da série B, neutralizando-se, assim, a diferença entre eles e os predicados descritivos:
24) $i \quad \emptyset$-to-ro mej
$1 \pm 3 \quad \mathrm{R}^{1}$-dançar-NLZ $\quad$ ser.bom
'eu dancei bem'
(Cabral, Rodrigues e Costa
[2001] 2003:27)
25) $i \quad \emptyset$-tẽ-m ket
$1 \pm 3 \quad \mathrm{R}^{1}$-ir/vir-NLZ neg
'eu não fui'
(Cabral, Rodrigues e Costa
[2001] 2003:27)

\footnotetext{
${ }^{8}$ Para Reis Silva (2001:19-20), os elementos de uma mesma série pronominal, os quais chamamos neste trabalho de formas pronominais da série $\mathrm{B}$, são tratados ora como marcas absolutivas, ora como marcas acusativas, como em ba a-kapreprek /1NOM 2AC-bater/ 'eu bati em você' e ba yre /1NOM cantar/ 'eu canto' (p.20). Em nossa análise essas formas pronominais são sempre marcas absolutivas.
} 
Cabral, Rodrigues e Costa ([2001] 2003:27) observam que o padrão ergativo-absolutivo do Xikrín é acionado em orações seguidas por advérbios ou por palavras ou orações modificadoras que qualificam ou negam o conteúdo do predicado. Assim, a cisão que distingue os alinhamentos nominativo-absolutivo e ergativo-absolutivo é condicionada pela modificação circunstancial dos predicados.

Acrescentamos a este o condicionamento sintático que requer nominalizações para funções nominais de núcleos verbais. Os autores observam que a cisão presente no Xikrín faz lembrar a cisão entre duas formas do modo indicativo, uma de expressão nominativa e a outra de expressão absolutiva, que ocorre nas línguas da família Tupí-Guaraní (Rodrigues 1953, Cabral 2001). Em ambos os casos há uma cisão de alinhamento não contemplada em ensaios sobre sistemas cindidos, como, por exemplo, em Dixon (1994).

\section{Correferencialidade interoracional em Xikrín}

Primeiramente esclarecemos que em Xikrín a estrutura de orações cuja tradução corresponde em outras línguas como o Português a subordinadas temporais ou condicionais se distingue de suas orações coordenadas, pela presença nas primeiras de partícula que pode ser traduzida por 'se' ou 'quando' e pela posição da oração dependente em relação à principal - nas temporais/ condicionais (hipotaxe) a dependente precede a principal, nas coordenadas (parataxe), a principal precede a dependente - como veremos adiante.

Em Xikrín, na combinação de duas orações por parataxe, se a oração principal e a oração dependente têm como núcleo predicados intransitivos e os seus sujeitos são correferentes, faz-se uso de duas estratégias: a) a combinação é marcada pela conjunção nẽe, que aqui glosamos de 'mesmo sujeito (MS)', e b) o sujeito da segunda oração é omitido:

$\begin{array}{lllllll}g a & n \tilde{e} & g a & a & \emptyset \text {-prõt- } \emptyset & \text { nẽ } & \emptyset \text {-boj- } \emptyset \\ 2 \pm 3 & \text { RLS } & 2 \pm 3 & 2 \pm 3 & \mathrm{R}^{1} \text {-corre r-NLZ } & \text { MS } & \mathrm{R}^{2} \text {-chegar-NLZ } \\ \text { 'você correu e chegou' (Costa, notas de campo 2008) }\end{array}$

A correferência configura-se como S/S. Eventualmente, pode ocorrer depois do conectivo de mesmo sujeito nẽ a posposição kam que contribui com a idéia de 'em relação a isso', 'assim', 'por isso'.

$\begin{array}{llllllll}g a & n \tilde{e} & g a & a & \emptyset \text {-prôt- } \emptyset & \text { nẽ } & \text { kam } & \emptyset \text {-boj- } \emptyset \\ 2 \pm 3 & \text { RLS } & 2 \pm 3 & 2 \pm 3 & \mathrm{R}^{1} \text {-corre r-NLZ } & \text { MS } & \text { por isso } & \mathrm{R}^{2} \text { chegar- NLZ } \\ \text { 'você correu e, por isso, chegou' (Costa, notas de campo } & 2008)\end{array}$


O mesmo padrão é encontrado em combinações em que a oração principal tem predicado com núcleo intransitivo e a dependente núcleo transitivo, evidenciando-se o padrão S/A:

28) memi nẽ boj nẽ mẽnire $\emptyset$-mũ

homem RLS chegar MS mulher $\mathrm{R}^{1}$-ver

'o homem chegou e viu a mulher' (Costa, notas de campo 2008)

Interessantemente, quando o predicado da oração dependente é negado, embora haja correferência, o S é obrigatoriamente marcado por proclítico pessoal combinado com o tema verbal, o que decorre da natureza da forma nominalizada do verbo, que exige um determinante expresso. Nesses casos, temos então um padrão em que o proclítico pessoal da oração dependente funciona como parte de um sistema de concordância com marca aberta de correferência.

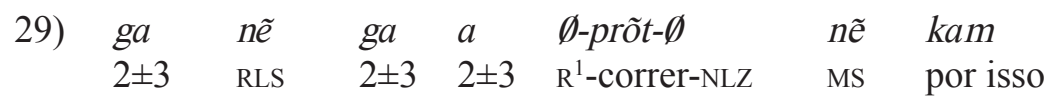

$\begin{array}{lll}a & \emptyset \text {-boj- } \emptyset & \text { ket } \\ 2 \pm 3 & R^{1} \text {-chegar-NLZ } & \text { NEG }\end{array}$

'você correu, mas não chegou' (Costa, notas de campo, 2008)

Nesse caso também, verifica-se um padrão nominativo de correferência $\mathrm{A} / \mathrm{S}$.

Quando a oração principal tem predicado cujo núcleo é um verbo transitivo e a segunda oração tem um predicado cujo núcleo é um verbo intransitivo, se os referentes de $\mathrm{Ae} \mathrm{S}$ são os mesmos, a correferência é marcada pela ausência de marcação de $\mathrm{S}$ na oração dependente, mantendo o padrão A/S. Entretanto, se a oração dependente for negada, S é obrigatoriamente marcado nesta última.

30) $b a \quad n \tilde{e} \quad b a \quad i \quad \emptyset$-bam $\emptyset$-mũ $\quad k a m \quad a k e \tilde{j}$

$1 \pm 3 \quad$ RLS $\quad 1 \pm 3 \quad 1 \pm 3 \quad \mathrm{R}^{1}$-pai $\quad \mathrm{R}^{1}$-ver $\quad \mathrm{MS} \quad$ voltar

'eu vi meu pai e voltei' (Costa, notas de campo, 2008)

31) $b a \quad n \tilde{e} \quad b a \quad i \quad \emptyset$-bam $\quad$-mũ $\quad k a m$

$1 \pm 3 \quad$ RLS $\quad 1 \pm 3 \quad 1 \pm 3 \quad \mathrm{R}^{1}$-pai $\quad \mathrm{R}^{1}$-ver $\quad \mathrm{MS}$

i j-akẽj ket

$1 \pm 3 \quad \mathrm{R}^{1}$-voltar $\quad \mathrm{NEG}$

'eu vi meu pai e não voltei' (Costa, notas de campo, 2008) 
$\begin{array}{llllll}\text { 32) } & \text { ne } & \text { bam } & \emptyset \text {-mũ } & \text { kam } & \emptyset \text {-akẽj } \\ \text { mãe } & \text { RLS } & \text { pai } & \mathrm{R}^{1} \text {-ver } & \text { MS } & \mathrm{R}^{2} \text {-voltar }\end{array}$

'a mãe viu o pai e voltou' (Costa, notas de campo, 2008)

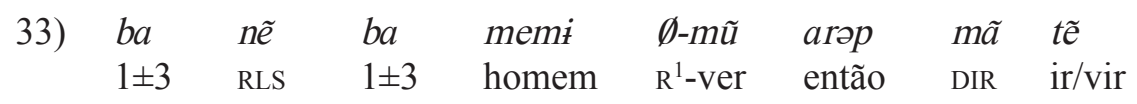

'eu vi o homem e, então, fui embora' (Costa, notas de campo, 2008)

Vimos até aqui que nas relações interoracionais a correferência sintática em Xikrín é possível entre S/S, S/A, A/S. Nessas combinações, verifica-se a presença da conjunção nẽ e a ausência da expressão de $\mathrm{S}$ ou $\mathrm{A}$ da oração dependente. Quando a combinação é A/S, nẽ não é usado, mas mantém-se a correferência marcada pela ausência de expressão de S na oração dependente, se esta não for negada ou modificada por outra expressão adverbial.

\subsection{Não-correferencialidade interoracional}

Na combinação de orações em que não há correferencialidade entre S e A, S e S e A/S, independentemente da transitividade do núcleo do predicado da oração dependente, a língua Xikrín dispõe das seguintes estratégias: a) as orações ocorrem justapostas e o sujeito da segunda oração é obrigatoriamente expresso, seja por pronome, seja por nome:

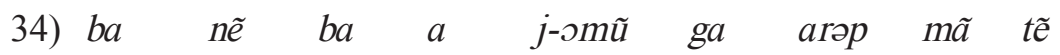
$1 \pm 3 \quad$ RLS $\quad 1 \pm 3 \quad 2 \pm 3 \quad \mathrm{R}^{1}$-ver $\quad 2 \pm 3 \quad$ PERF $\quad$ DIR $\quad$ ir/vir 'eu vi você e você foi embora' (Costa, notas de campo, 2008)

35) memi na boj ga a-je $\emptyset$-smũ-j ket homem REAL $2 \pm 3 \quad 2 \pm 3 \quad 2 \pm 3$-ERG $\quad R^{2}$-ver-NLZ NEG 'o homem chegou, mas você não o viu' (Costa, notas de campo, 2008)

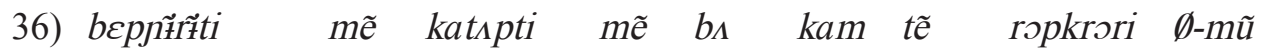
Bep Nhõrõ-Ti cons Katop-Ti cons mata posp ir/vir onça $\mathrm{R}^{1}$-ver 'Bep Nhõrõ-Ti e Katop-Ti foram caçar e viram uma onça' (Costa, notas de campo 2008)

b) as duas orações são combinadas pela conjunção um e o sujeito da segunda oração pode ou não ser expresso se for diferente de $\mathrm{S}$ ou $\mathrm{A}$, mas igual a $\mathrm{O}$ da primeira oração:

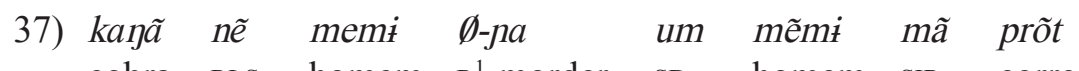
cobra RLS homem $\mathrm{R}^{1}$-morder SD homem SIR correr 'a cobra mordeu o homem e o homem correu' (Costa, notas de campo, 2008) 
38

$\begin{array}{llllllll}n \tilde{a} & \text { ne } & \text { bam } & \emptyset \text {-mũ } & \text { um } & \text { kam } & \text { bam } & \text { akẽ j } \\ \text { mãe } & \text { RLS } & \text { pai } & \mathrm{R}^{1} \text {-ver } & \text { SD } & \text { então } & \text { pai voltar }\end{array}$

$\begin{array}{lllllll}\text { 39) } & \text { ñ } & \text { bam } & \emptyset \text {-mũ } & \text { um } & \text { bam } & a k \tilde{e} j \\ \text { mãe } & \text { RLS } & \text { pai } & \mathrm{R}^{1} \text {-ver } & \text { SD } & \text { pai } & \text { voltar }\end{array}$

'a mãe viu o pai e voltou' (Costa, notas de campo, 2008)

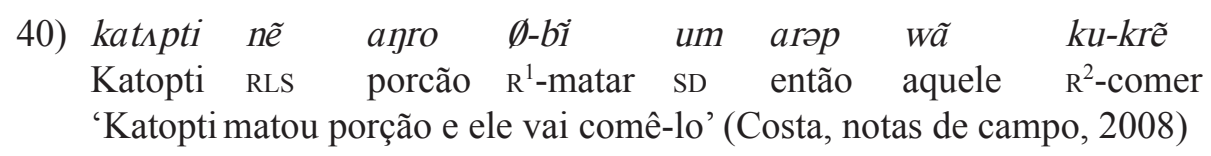

No exemplo (38), bam pode ser expresso porque não é correferencial com o referente do sujeito da oração principal. Em (39), ocorre o mesmo padrão, mas sem o morfema kam; em (40), wã marca uma terceira pessoa, diferente de Katopti e de ayro. O sujeito dessa oração não poderia ser correferente com o referente do agente da primeira oração por causa da conjunção $u m$, que marca referência disjunta e, se fosse correferente com ayro, ou ayro seria expresso ou seria omitido.

No exemplo seguinte, embora um marque a não correferência de $\mathrm{S}$ da oração dependente com A da oração principal, S não é marcado, pois é correferente com o objeto da oração principal:

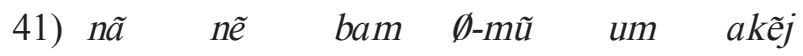

mãe RLS pai $\mathrm{R}^{1}$-ver $\mathrm{SD}$ voltar

'a mãe viu o pai e (o pai) voltou' (Costa, notas de campo, 2008)

42) $b a \quad n \tilde{e} \quad b a \quad i \quad \emptyset$-bam

$1 \pm 3 \quad$ RLS $\quad 1 \pm 3 \quad 1 \pm 3 \quad \mathrm{R}^{1}$-pai $\quad \mathrm{R}^{1}$-ver $\quad \mathrm{SD} \quad$ correr

'eu vi meu pai e (ele) correu' (Costa, notas de campo, 2008)

Os dois últimos exemplos mostram que a língua Xikrín faz uso também de um sistema de correferência $\mathrm{O} / \mathrm{S}$. A coocorrência de um sistema nominativo e de um sistema absolutivo faz do sistema de correferência do Xikrín um sistema misto, que é uma possibilidade nas línguas naturais, como observado por Dixon (1994:154), de forma que o monitoramento de correferência é um monitoramento complexo e bidirecional.

No exemplo seguinte, $\mathrm{S}$ da oração principal é correferente com $\mathrm{O}$ da oração dependente, mas nada de especial é marcado, apenas a não correferência entre $\mathrm{S}$ e A. 
43) $\begin{array}{llllllll}b a & n \tilde{e} & b a & \text { boj } & \text { um } & \text { memi } & i & j \text {-omũ } \\ 1 \pm 3 & \text { RLS } & 1 \pm 3 & \text { chegar } & \text { SD } & \text { homem } & 1 \pm 3 & \mathrm{R}^{1} \text {-ver } \\ \text { 'eu cheguei e o homem me viu' (Costa, notas de campo, 2008) }\end{array}$

Quando duas orações expressam processos excludentes, S ou Aé marcado em todas as orações, mesmo que haja correferência, visto que semanticamente não há relação entre os referentes dos argumentos das duas orações:

44) $\quad g a \quad d z a \quad g a \quad m a r a b a \quad \emptyset-m a ̃ \quad$ tẽ norokot dza $g a$ $2 \pm 3$ IRLS $2 \pm 3$ Marabá $R^{1}$-POSP ir/vir ou

parawapebas $\emptyset$-mã tẽ

Parauapebas $\quad \mathrm{R}^{1}$-POSP $\quad$ ir/vir

'ou você vai a Marabá ou você vai a Parauapebas'

(Costa, notas de campo, 2008)

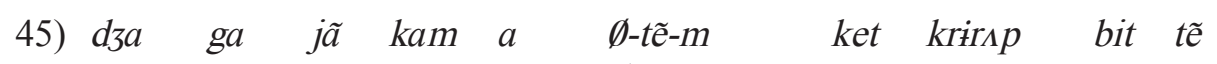
IRLS $2 \pm 3$ esse LOC $2 \pm 3 \quad \mathrm{R}^{1}$-ir/vir-NLZ NEG amanhã só ir/vir

$\begin{array}{lllll}\text { norokot } & \tilde{o} & \mathrm{kam} & \text { bit } & \text { tẽ } \\ \text { ou } & \text { outro } & \text { LOC } & \text { só } & \text { ir/vir }\end{array}$

'se você não for para a aldeia hoje, você irá outro dia' (Costa, notas de campo, 2008)

Já em combinações em que uma delas exprime hipótese, o que se faz por meio do morfema $d z a$ (dependência semântica que é expressa por meio de processos de hipotaxe em outras línguas) em posição inicial da sentença, havendo correferência entre $\mathrm{A} / \mathrm{S}$ ou $\mathrm{S} / \mathrm{A}, \mathrm{S}$ ou $\mathrm{A}$ da oração principal não é marcado, visto que a oração dependente a precede. Isto evidencia que o princípio da anaforicidade prevalece nesta língua.

46) d3a $_{3}$ ga maraba Ø-mã tẽ-m ket nẽ kam

IRLS $2 \pm 3$ Marabá R ${ }^{1}$-POSP ir/vir-NLZ NEG MS então

parawapebas Ø-mã tẽ

Parauapebas $\mathrm{R}^{1}$-POSP ir/vir

'se você não for para Marabá você irá para Parauapebas'

(Costa, notas de campo, 2008)

47) $\quad$ d3a $\quad g a \quad j \tilde{a} \quad k a m$ a IRLS $2 \pm 3$ esse LOC $2 \pm 3$ R ${ }^{1}$-ir-NLZ NEG amanhã só ir/vir ou 
$\begin{array}{llll}\tilde{o} & \mathrm{kam} & \text { bit } & \text { tẽ } \\ \text { outro } & \text { LOC } & \text { só } & \text { ir/vir }\end{array}$

'se você não for para a aldeia hoje, você irá outro dia'

(Costa, notas de campo, 2008)

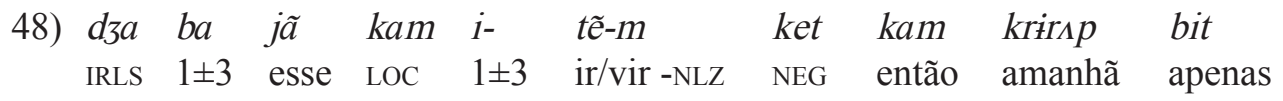

bit tẽ norokst $\tilde{o}$ kam bit tẽ

apenas ir ou outro LOC apenas ir/vir

'se eu não for hoje, irei amanhã ou irei outro dia'

(Costa, notas de campo, 2008)

\section{Correferencialidade em outras línguas Jê}

Urban (1985) mostra que em Xokléng é o uso do pronome especial $\tilde{\varepsilon}$, forma pronominal anafórica - ou mais frequentemente catafórica -, que indica correferência entre argumentos; já o uso de pronomes nãocorreferenciais indica referência disjunta. Urban (op.cit.) nota, ainda, que em Xokleng o pronome $\tilde{\varepsilon}$ é usado em oração subordinada para indicar que o sintagma nominal que ele substitui tem o mesmo referente que um sintagma nominal da oração principal. Segundo esse autor, se o correferente do sintagma nominal da oração principal está em função de $\mathrm{S}, \tilde{\varepsilon}$ pode ser usado para substituir S, A, ou O na oração subordinada, como em (63), (65), e (66), respectivamente (a numeração original dos exemplos foi mantida):

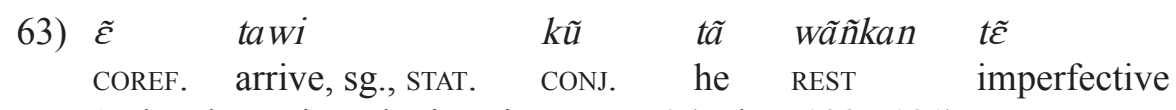

'When he ${ }_{i}$ arrives, he $_{i}$ is going to rest.' (Urban 1985:181)

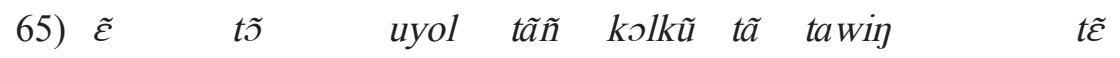
COREF. ergative tapir kill after he arrive, SG., ACT. imperfective 'After he ${ }_{\mathrm{i}}$ kills the tapir, he ${ }_{\mathrm{i}}$ is going to arrive.' (Urban 1985:181)

66) to $\quad \begin{array}{lllllllll} & \delta i & t \tilde{s} & \tilde{\varepsilon} & w e & k o l k u & t \tilde{a} & w \tilde{u}\end{array}$ woman FEM. ergative COREF. See, STAT. after he 3-NOM

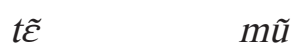
go, sg., act. active

'After the woman saw himi, hei left.' (Urban 1985:181) 


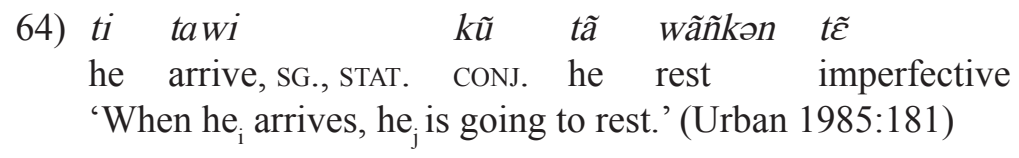

Ainda de acordo com Urban (op.cit.), quando o correferente do sintagma nominal da oração principal está em função de A, $\tilde{\varepsilon}$ é usado, mas apenas quando correferente com S ou A da oração subordinada, como mostram os exemplos em (67) e (68):

67)
$\tilde{\varepsilon} \quad$ tawi
$k \tilde{u} \quad t a \tilde{a}$
a $a$

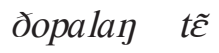
COREF. arrive, SG., STAT CONJ. he you teach imperative

'when he ${ }_{i}$ arrives, he ${ }_{i}$ is going to teach you.' (Urban 1985:181)

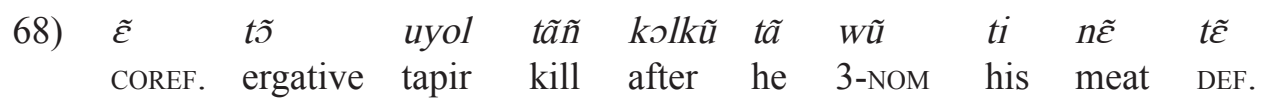

ko t $\tilde{\varepsilon}$

eat imperfective

'After he kills the tapir he is going to eat its meet.'(Urban 1985:182)

Se o correferente do sintagma nominal da oração subordinada estiver em uma função de $\mathrm{O}, \tilde{\varepsilon}$ não é usado, mas uma terceira pessoa $t i$ ou $z i$ :

69)

\begin{tabular}{|c|c|c|c|c|c|c|}
\hline $\begin{array}{l}\text { to } \\
\text { woman }\end{array}$ & $\begin{array}{l}\partial i \\
\text { FEM. }\end{array}$ & $\begin{array}{l}t \tilde{3} \\
\text { ergative }\end{array}$ & $\begin{array}{l}t i \\
\text { he }\end{array}$ & $\begin{array}{l}\text { we } \\
\text { see, STAT. }\end{array}$ & $\begin{array}{l}\text { kolkũ } \\
\text { after }\end{array}$ & $\begin{array}{l}\text { tã } \\
\text { he }\end{array}$ \\
\hline & $p \tilde{a} \eta$ & & $m \tilde{u}$ & & & \\
\hline
\end{tabular}

'After the woman saw him, he , $_{\mathrm{i}}$ shot (at) her.' (Urban 1985:182)

Urban (op.cit.) conclui que o sujeito sintático nesta língua não é claramente definido, visto que $\tilde{\varepsilon}$ pode ser usado também para um $O$ da oração subordinada correferente com $\mathrm{S}$ da oração principal. Para esse autor, se não fosse por isso, o padrão do Xokléng corresponderia a um simples sistema de switch-reference, com um sujeito sintático definido em termos da confluência de A e S. Como ressalta Urban (op.cit.), o Xokléng mostra nesta área da sintaxe um padrão claro, mas que não é nem acusativo nem ergativo.

Outras línguas Jê para as quais o fenômeno switch-reference foi descrito são o Crahô (Popjes \& Popjes 1986), o Parkatêjê (Ferreira 2003) e o CanelaApãniekrá (Alves 2004). 
Popjes \& Popjes (1986) mostram que o Canela-Krahô dispõe de morfemas independentes para indicar o fenômeno de switch-reference entre sujeitos de duas ou mais orações: a conjunção ne, dentre outras funções que desempenha na trama sintática da língua, é usada para indicar que sujeitos de duas ou mais orações são correferentes, conforme ilustra o exemplo (49):

49) Capi te pó curan ne quê há cuku Capi PAST deer kill and 3 FUT 3+eat

'Capi killed a deer and will eat it' (Popjes \& Popjes 1986:147)

Para marcar a não-correferencialidade dos sujeitos, as orações são ligadas pela conjunção $m \tilde{a}$, como exemplificado em (50):
50) a-te pó curan mã capi Apu cuku
2-PAST deer kill and Capi CONT 3+eat
'You killed a deer and Capi is eating it.' (Popjes \& Popjes 1986:147)

Na língua Parkatêjê, o fenômeno switch-reference é marcado por dois morfemas independentes, a conjunção ñ̃, usada para indicar que sujeitos de duas orações têm o mesmo referente, e a conjunção mõ, utilizada para sinalizar que os sujeitos de duas orações são diferentes (cf. Ferreira 2003). Os exemplos (51) e (52), extraídos de Ferreira (2003:183) exemplificam sujeitos correferentes e sujeitos não-correferentes, respectivamente.
51) int Jũm te mïre koran ñ̃ te kukrit pïr meu. Pai ERG jacaré matar SS ERG anta matar+ PAS 'meu pai matou jacaré e matou anta' (Ferreira 2003:183)

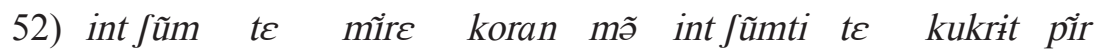
meu.pai ERG jacaré matar DS meu.tio ERG anta ONC-matar+PAS 'meu pai matou jacaré e meu tio também matou anta' (Ferreira 2003:183)

Alves (2004:143) descreve duas estratégias de que a língua Canela Apãniekrá dispõe para marcar a correferência dos sujeitos em sentenças coordenadas, o apagamento do mesmo SN sujeito da segunda oração e uso da conjunção $n \tilde{\varepsilon}$, como ilustra o exemplo (53).

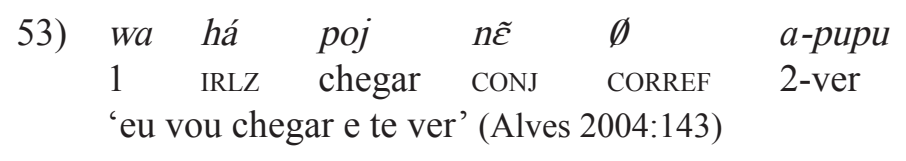


Alves (op. cit. p. 143) observa que quando há um padrão ergativo, "ainda que seja possível, não é comum apagá-lo, e que, nesses casos, só ocorre a conjunção":

53) $\begin{array}{llll}i-p o j & n \tilde{\varepsilon} & i-t \varepsilon & \text { a-pupu } \\ 1 & \text { CONJ } & 1 \text {-ERG } & 2 \text {-ver }\end{array}$

'eu cheguei e vi você' (Alves 2004:143)

Já, para marcar que o sujeito da oração principal não é correferente com o da oração dependente, o Apãniekrá utiliza a conjunção mãa, conforme mostra o exemplo (54).

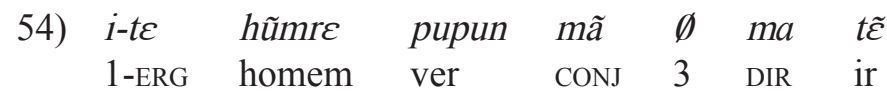

'eu vi o homem e ele foi embora' (Alves 2004:144)

Alves observa que quando S1 e S2 são nominais, S2 não é apagado:

55) Pedro te João pupun mã João mor

Pedro RG João ver CONJ João ir

'Pedro viu João e João foi embora' (Alves 2004:144)

A autora descreve outras situações de expressão de correferência na língua, orientando sua descrição para preocupações de outra natureza: a origem do fenômeno de switch-reference na língua, e a descrição tipológica desse sistema (...) "dentro do domínio funcional da continuidade do tópico no discurso" (...) (cf. p. 157).

Maxakalí, da família do mesmo nome, é outra língua na qual switchreference foi identificado. Rodrigues (1999:197-198) mostra que o Maxakalí utiliza a conjunção ti para marcar a correferencialidade dos sujeitos de duas ou mais orações, ao passo que a conjunção $h a$ distingue orações com sujeitos diferentes, como mostram os exemplos (55) e (56) respectivamente.

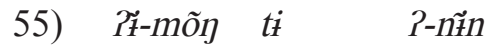

3-go and.ss 3-come

'He went and returned' (Rodrigues 1999:197)

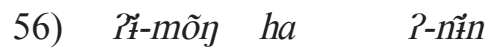

3-go and.DS 3-come

'He went and he returned' (Rodrigues 1999:198)

É interessante notar que não só as línguas da família Jê setentrional e meridional apresentam um sistema de referência alternada que distingue 
mesmo sujeito de sujeito diferente, mas que o mesmo fenômeno é encontrado em membro de outra família Macro-Jê, o Maxakalí, o que sugere que um sistema dessa natureza tenha estado ativo em estágio anterior comum a essas e outras famílias incluídas na proposta de um Macro-Jê por Rodrigues (1986, 1992, 1996, 2001, 2009).

Por outro lado, a exploração que fizemos dos dados do Xikrín revela que, embora nessa língua haja um sistema de referência alternada, com marcas de mesmo sujeito e sujeito diferente, os quais contribuem para a coesão discursiva e comunicação plena, este sistema é mais sofisticado do que uma simples alternância de correferência fundada em uma orientação S/A (nominativa). Na seção seguinte resumiremos as características do sistema de correferencialidade do Xikrín.

\section{Algumas observações sobre correferencialidade sintática em Xikrín}

De acordo com os dados apresentados, pode-se concluir que na língua Xikrín há um sistema de referência-alternada, sinalizado por partículas e por omissão ou não da expressão sintática de argumentos correferentes com argumentos da oração principal. A presença de uma estratégia ou de outra, depende de fatores pragmáticos e de princípios gramaticais que interagem com os princípios que determinam alinhamento, seja no nível intraoracional, seja no nível interoracional.

Nas combinações de orações por parataxe, as partículas comportamse como conjunções conectivas e não como morfemas subordinadores sistematicamente recorrentes, como ocorre em outras línguas indígenas brasileiras, como as da família Tupí-Guaraní. Em Xikrín, são conectivos exclusivos de orações, pois o conectivo de nomes que formam um sintagma nominal tem a forma mẽ. $\mathrm{O}$ uso das partículas não é sistemático, já que paralelamente ao seu uso, a língua pode se valer da omissão ou expressão de argumentos para monitorar correferência entre estes, em determinados contextos sintáticos.

As combinações de orações por parataxe em Xikrín seguem uma ordem canônica: a oração em que os referentes são obrigatoriamente expressos é a primeira, sendo a segunda aquela que segue regras morfossintáticas de correferência. A segunda oração pode manter certa dependência semântica da primeira, como em Português: 'eu descasco o camarão e você o tempera', 'eu pesco o peixe e você o come'. 
Diferentemente da parataxe, nas combinações por hipotaxe, a primeira oração é semanticamente dependente da segunda, o que é exigido pelo princípio da anaforicidade que determina correferência na língua:

- Elementos acionadores de correferência através de fronteiras de orações devem ter precedência sintática.

Os pivots da correferência interoracional em Xikrín são A e S, mas há sintonia desta correferência com a correferência entre $\mathrm{O}$ e $\mathrm{S}$, quando o contexto morfossintático exige. Esta sintonia ocorre na situação em que duas orações têm sujeitos distintos e o objeto da primeira é correferente com o sujeito da oração dependente. Neste caso, S não é marcado, embora as duas orações tenham sujeitos diferentes, o que revela que, além do alinhamento sintático nominativo, A/S,S/A, o Xikrín alinha $\mathrm{O} / \mathrm{S}$, um padrão absolutivo. Nesse sentido, o Xikrín se assemelha ao Xokléng, conforme a descrição de Urban (1986:183):

However, in Shokleng the syntactic "subject" is not so clearly defined. This is because E may be used for subordinate clause $\mathrm{O}$, when this noun phrase is referent with main clause $\mathrm{S}$. If this were not the case, the pattern would be one of ordinary "switchreference", with a syntactic "subject" defined in terms of the conflation of A and S. Since it is, however, Shokleng shows in this area of syntax a clear pattern, but one that is neither accusative nor ergative in its entirety.

Correferencialidade em Xikrín é marcada entre duas orações, estejam elas internamente estruturadas ou não de acordo com o mesmo sistema nominativo-absolutivo ou o mesmo sistema ergativo-absolutivo. A particularidade da marcação de correferência em construções ergativasabsolutivas é que nelas há sempre uma expressão sintática de argumentos, o que a diferencia de construções nominativas-absolutivas nas quais, havendo correferência, os argumentos A e S podem não ser expressos.

Sumarizando, a língua Xikrín possui um sistema de referência alternada que faz uso de marcas morfológicas concomitantemente ou não com a expressão ou omissão de argumentos sintáticos, de acordo com necessidades pragmáticas e com princípios da sintaxe da língua.

Os resultados obtidos até o presente reiteram que o Xikrín é uma língua que apresenta cisões em seu sistema de alinhamento intraoracional, ergativo-absolutivo e nominativo-absolutivo (Cabral, Rodrigues e Costa [2001] 2003; Costa 2003), e mostram que a correferencialidade através 
de orações também apresenta cisões: por um lado, a correferência segue um alinhamento A e S ou S e A, mas, por outro lado, um alinhamento $\mathrm{O}$ e S. Resta confirmar se esses padrões de correferência se estendem a outras combinações de orações.

Finalmente, no aprofundamento deste estudo, deverão ser consideradas diferentes modalidades discursivas, para que apreciações sobre a caracterização do sistema de correferência em Xikrín, associado a alinhamento, sejam conclusivas.

\section{Referências}

Alves, F. C. 2004. O Timbira falado pelos Canela Apãniekrá: uma contribuição aos estudos da morfossintaxe de uma língua Jê. Tese de doutorado, Universidade Estadual de Campinas.

Costa, L. S. C. 2003. Flexão relacional, marcas pessoais e tipos de predicados em Xikrín: contribuição para os estudos sobre ergatividade em línguas Jê. Dissertação de mestrado, Universidade Federal do Pará.

Cabral, Ana Suelly A. C., A. D. Rodrigues, L. S. da Costa. 2003. Notas sobre ergatividade em Xikrín. Liames, 4:21-28.

Dixon, R. M. W. 1994. Ergativity. Cambridge: Cambridge University Press.

Foley, W. A., Robert D. Van Valin. 1984. Functional syntax and universal grammar. Cambridge: Cambridge University Press.

Ferreira, M. N. O. 2003. Estudo morfossintático da língua Parkatêjê. Tese de doutorado, Universidade Estadual de Campinas.

Huang, Y. 2003. Switch-reference in Amele and logophoric verbal suffix in Gokana: a generalized neo-Gricean pragmatic analysis. Reading Working Papers in Linguistics 7:53-76.

Popjes, J., J. Popjes. 1986. Canela-krahô. In: D. C. Derbyshire, G. K. Pullum (eds.) Handbook of Amazonian languages 1:128-199. Berlin, New York, Amsterdam: Mouton de Gruyter.

Rodrigues, Aryon D.1986. Línguas brasileiras: para o conhecimento das linguas indígenas. São Paulo: Edições Loyola.

Rodrigues, Aryon D. 1999. Macro-Jê. Robert M. W. Dixon, Alexandra Y. Aikenvald (eds.). Amazonian languages. Cambridge: Cambridge University Press.

Rodrigues, Aryon D. 2001. Flexão relacional no tronco linguístico Macro-Jê. Boletim da Abralim, 25:219-231. 
Rodrigues, Aryon D. 2009. A case of affinity among Tupí-Karíb and Macro-Jê. Revista Brasileira de Linguística Antropológica 1.2:137-162.

Shachter, P. 1974. A non-transformational account of serial verbs. Studies in African Linguistics, Supplement 5:252-70.

Urban, G. 1985. Ergativity and accusativity in Shokleng. International Journal of American Linguistics, 51.2:164-187. 